$63^{\text {ème }}$ Congrès de la SFCO, 03013 (2015)

DOI:10.1051/sfco/20156303013

(C) Owned by the authors, published by EDP Sciences, 2015

\title{
Granulome pyogénique et implants dentaires : à propos d'un cas
}

\author{
Millot $\mathrm{G}^{*}$, Myara $\mathrm{M}^{* *}$, Pinto $\mathrm{A}^{* * *}$, Alantar $\mathrm{A}^{* * * *}$ \\ * Interne Chirurgie Orale, Région Ile-de-France \\ ** Praticien attaché Hôpital Max Fourestier, Service d'Odontologie, Nanterre \\ *** Exercice libéral Paris \\ **** Praticien Hospitalier, Hôpital Max Fourestier, Service d'Odontologie, Nanterre
}

Un homme de 62 ans sans antécédents médicaux est adressé en consultation de chirurgie orale pour une lésion gingivale péri-implantaire évoluant depuis plusieurs mois.

Le patient ne présente pas d’altération de l'état général, il décrit une simple gêne.

La palpation cervicale révèle une adénite sous-mandibulaire homolatérale. A l'examen endo-buccal, le patient présente une parodontite chronique généralisée, associée à de nombreuses restaurations prothétiques dento-portées et implanto-portées.

La lésion a l'aspect d'un nodule hyperplasique bilobé, pédiculé et rougeâtre, située sur la gencive de part et d'autre des implants 34/35/36/37. Elle est ulcérée en surface, de consistance molle et mesurant $15 \mathrm{~mm}$ dans son grand axe. On note un saignement au contact et une profondeur au sondage de $5 \mathrm{~mm}$ autour des implants en site de 35 et 36.

On observe sur l'orthopantomogramme une lyse osseuse péri-implantaire en cupule permettant d'évoquer le diagnostic de péri-implantite associé un épulis inflammatoire ulcéré. Le diagnostic différentiel peut se faire avec un carcinome épidermoïde (saignement au contact, adénopathie).

Après biopsie, l'examen anatomo-pathologique révèle un « aspect histologique de bourgeon charnu hyperplasique et hypervascularisé, recouvert d'un enduit fibrinoleucocytaire et riche en polynucléaires, en faveur d'un granulome pyogénique ulcéré ». A la suite de l'enseignement des techniques d'hygiène orale et la prescription de brossettes inter-dentaires, une exérèse large de la lésion et un surfaçage des implants à l'aide d'une curette en plastique sont alors réalisés, puis un pansement Coe-Pack ${ }^{\circledR}$ est mis en place en vestibulaire et lingual. Un traitement parodontal est mené afin de stabiliser la parodontite. Le contrôle à 2 mois montre une bonne cicatrisation et les séances de maintenance parodontale et implantaire contribuent à prévenir les récidives.

Le granulome pyogénique (botryomycome, bourgeon charnu hyperplasique ou hémangiome capillaire lobulé) est une lésion bénigne d’origine vasculaire (Fortna et al. 2007) dont la prévalence n’est pas précisée dans la littérature. Il siège sur la peau et les muqueuses, en particulier sur les lèvres, les gencives, les joues et la langue. Souvent unique mais parfois multiple (Shah et al. 1995), il se développe le plus fréquemment à partir d'une ulcération, d'un traumatisme, d'une petite plaie, d'une irritation chronique ou d'aspérités secondaires à des soins dentaires (Jafarzadeh et al. 2006, Sarvana et al. 2009).

Le développement d'un granulome pyogénique gingival peut aussi être en rapport avec les modifications hormonales des femmes enceintes (Henry et al. 2006).

Quelques cas péri-implantaires sont décrits dans la littérature. Outre les causes traumatiques et hormonales, une irritation chronique due à la corrosion du titane pourrait entraîner le développement de cette tumeur bénigne (Kang et al. 2014). En effet, il existe un lien entre la réponse inflammatoire péri-implantaire et la présence d'ions et de particules métalliques contenus dans les tissus adjacents aux implants ayant subi un processus de corrosion (Olmedo et al. 2008, Olmedo et al. 2010).

This is an Open Access article distributed under the terms of the Creative Commons Attribution License 4.0, which permits unrestricted use, distribution, and reproduction in any medium, provided the original work is properly cited. 
Le traitement de choix de ces lésions est l'exérèse chirurgicale large, pour diminuer les risques de récidives, et de préférence à la lame froide assurant un examen anatomo-pathologique fiable (Wauters et al. 2010).

La prévention des péri-implantites est au coeur des préoccupations actuelles en implantologie, l'importance de la maintenance implantaire, prothétique et parodontale est capitale dans le suivi des patients.

Nom et adresse du conférencier

Gaël MILLOT

Hôpital Max Fourestier

403, avenue de la République

92000 Nanterre (France)

millotgael@hotmail.fr 